\title{
Differential chromosome pairing affinities at meiosis in polyploid sugarcane revealed by molecular markers
}

\author{
N Jannoo ${ }^{1}$, L Grivet ${ }^{1}$, J David ${ }^{2}$, A D'Hont ${ }^{1}$ and J-C Glaszmann ${ }^{1}$ \\ ${ }^{1}$ CIRAD, UMR 1096, TA 40/03, Avenue Agropolis, 34398 Montpellier Cedex 5, France; ${ }^{2}$ INRA, UMR 1097, Domaine de Melgueil, \\ 34130 Maugio, France
}

\begin{abstract}
Chromosome pairing at meiosis is an essential feature in cell biology, which determines trait inheritance and species evolution. Complex polyploids may display diverse pairing affinities and offer favorable situations for studying meiosis. The genus Saccharum encompasses diverse forms of polyploids with predominantly bivalent pairing. We have focused on a modern cultivar of sugarcane, R570, and taken advantage of a particular single copy probe (BNL 12.06) revealing 11 alleles by restriction fragment length polymorphism (RFLP). As for other cultivars, R570 is highly polyploid $(2 n=$ ca. 115$)$ and indirectly derived from interspecific hybridization between Saccharum officinarum $(2 n=80$, $x=10)$ and $S$. spontaneum $(2 n=40-128, x=8)$. Here we determined the doses of the various BNL12.06 RFLP alleles
\end{abstract}

among 282 progeny of $\mathrm{R} 570$ and estimated the mutual pairing frequencies among the corresponding homo- or homoeologous chromosomes using a maximum likelihood method. The result is an atypical picture, with pairing frequencies ranging from 0 to $40 \%$ and differential affinities leading to the identification of several chromosome subsets. This example illustrates the unsystematic meiotic behavior in a complex polyploid. It highlights a continuous range of pairing affinities between chromosomes and pinpoints a strong role of individual chromosome features, partly related to their ancestral origin, in the determination of these affinities.

Heredity (2004) 93, 460-467. doi:10.1038/sj.hdy.6800524

Published online 4 August 2004

Keywords: chromosomal pairing; meiosis; polyploid; Saccharum; RFLP

\section{Introduction}

Polyploidy, that is the occurrence of more than two copies of the basic set of chromosomes in a somatic cell, is very common in plants. While it has long been accepted that as many as $50 \%$ of the plant taxa are polyploid or derived from ancient polyploidy (Stebbins, 1971), recent studies on sequence conservation in Arabidopsis thaliana indicate that even the simplest genomes exhibit traces of past polyploid history (Blanc et al, 2000; Vision et al, 2000). Rounds of polyploidization, by addition of several genomes, followed by 'diploidization' and stabilization of meiotic features leading to pairs of homologous chromosomes have been a major component of genome evolution in plants. Chromosome pairing at meiosis is well determined in diploids, where each chromosome consistently finds its homologous counterpart to give rise to disomic segregation. Polyploids display a wider array of situations. Whether meiosis yields bivalent (by pair) or multivalent (involving more than two) chromosome associations at metaphase I of meiosis affects the pattern of chromosomal segregation to daughter cells, as well as the opportunities for chromosomal interchanges by chiasma formation. When

Correspondence: J-C Glaszmann, CIRAD, UMR 1096, TA 40/03, Avenue Agropolis, 34398 Montpellier cedex 5, France.

E-mail: jean-christophe.glaszmann@cirad.fr

Received 17 December 2003; accepted 18 May 2004; published online 4 August 2004 only bivalents are formed, the way the chromosomes pair can vary between full predetermination, producing disomic inheritance and random association, leading to polysomic inheritance. The process that governs chromosome pairing at meiosis is not well understood. It involves specific genetic factors, such as the Ph1 gene in wheat (Riley and Chapman, 1958; Martinez-Perez et al, 2001), as well as structural features (Jenkins and Chatterjee, 1995). Modern sugarcane cultivars constitute a particularly complex case of polyploidy, as described by Grivet and Arruda (2001). They originate from recombination among hybrid derivatives between two highly polyploid species, namely Saccharum officinarum $(x=10,2 n=8 x=80)$ and $S$. spontaneum $(x=8,2 n=$ $5-16 x=40-128)$. They usually display between 100 and 130 chromosomes (70-80\% appear derived from S. officinarum), while 10-20\% appear derived from $S$. spontaneum and about $10 \%$ are recombinants of both origins (D'Hont et al, 1996). The genetic maps available so far suggest that the parental genomes are collinear and probably differ by only a small number of rearrangements (Grivet et al, 1996; Ming et al, 1998).

Despite the high ploidy level, both parental species mainly form bivalents at meiosis (Bremer, 1929; Sreenivasan, 1975). In interspecific cultivars, meiotic metaphase spreads reveal swarms of chromosomes that are difficult to interpret, but show mostly bivalents. The most detailed studies of pollen mother cells in a range of cultivars (Price, 1963; Burner, 1994) report less than one multivalent (tri- or quadrivalent) and three to four 
univalents per cell on average. New insights into meiosis were brought about by molecular marker segregations, which allow the identification of chromosome-pairing affinities, thanks to linkages in the repulsion phase. This approach, although based on partial genome coverage, suggested polysomic inheritance in studies conducted using progeny from S. spontaneum (Al-Janabi et al, 1993; Da Silva et al, 1993), on the basis of a general lack of linkage in repulsion. Studies that used an S. officinarum parent detected some instances of repulsion, indicative of preferential pairing between a subset of chromosomes (Al Janabi et al, 1994; Ming et al, 1998). The first segregation studies conducted in a cultivar, namely R570, were based on RFLP among 77 individuals derived from selfpollination (Grivet et al, 1996). They led to the identification of 99 unsaturated linkage groups that covered approximately one-third of the whole genetic map. These linkage groups were gathered into 10 tentative homology classes (HC) on the basis of probes in common, enabling the identification of sets of homologous chromosomes. Several instances of repulsion were detected, essentially between $S$. spontaneum-derived chromosomes. A recent study using 295 individuals of the same self-progeny monitored for close to a thousand segregating amplified fragment length polymorphism (AFLP) bands (Hoarau et al, 2001) ensured half coverage of the genetic map. Out of 120 linkage groups and 52 unlinked markers, the repulsions highlighted 12 couples of chromosomes that are strongly associated at meiosis. At least two of these couples apparently display systematic pairing. The chromosomes involved appeared to be of mixed origin, and several instances of pairing between regions derived from the two ancestral species were detected. These mapping studies provided instructive features, but had several drawbacks for an accurate description of chromosome pairing. They relied on markers scored as dominant with no insight into allele dosage, which limited the accuracy of genotype determination. They also generally failed to cover all homologues in a given $\mathrm{HC}$, for only chromosomes bearing single dose alleles were detected. This allowed only incomplete survey among the interacting chromosomes.

We describe a particular approach based on RFLP analysis using a particular probe chosen, because it enables us to access all chromosomes of a given $\mathrm{HC}$ and to characterize their segregation taking account of their dosage. This provides a unique description of chromosome assortment in what is probably the most complex crop genome studied to date.

\section{Materials and methods}

The plant materials used were chosen from those used and described by Hoarau et al (2001). They consisted of
282 individuals derived from the selfing of cultivar 'R570' $(2 n=$ ca. 115), an important commercial variety.

RFLP analysis was performed with standard procedures, as described in Grivet et al (1996). The individual plant DNA was cut separately with three restriction enzymes and was subjected to Southern analysis using BNL12.06 as a probe. Those bands that segregated in a 3:1 (presence:absence) fashion within the progeny were scored; they are single-dose restriction fragments (SDRF) (Wu et al, 1992) in the parental cultivar. The similarity in segregation of two SDRF revealed with two different restriction enzymes is indicative of an allele that can be resolved by both enzymes. The use of three restriction enzymes ensured some redundancy in the detection of several alleles, which was useful for data quality control. A total of 11 alleles, numbered from 1 to 11 , could be identified. They correspond to the 11 alleles detected by Grivet et al (1996); their resolution by the various restriction enzymes is given in Table 1 . These alleles belong to 11 linkage groups (Figure 1), of which 10 are homologous (or homoeologous when inherited from $S$. officinarum and S. spontaneum), for they share markers derived from the same series of RFLP probes. The 11th one comprises the BNL12.06 marker, but its other constitutive markers belong to another set of homologous linkage groups. The possibility that additional alleles exist other than the 11 scored could be discarded for two main reasons. Firstly, all the segregating bands that corresponded to multiple-dose restriction fragments could be accounted for by various combinations of the 11 alleles. Secondly, among the eight probes that constitute HC I, none revealed any unlinked marker that would suggest the existence of other, poorly marked, chromosomes of this HC (Grivet et al, 1996). Therefore, it is considered that all the chromosomes of HC I are covered

The analysis of chromosome assortment took account of the massive predominance of bivalents observed in all Saccharum forms, including cultivars. Given that the SDRF are present as a single dose in the parent, the dosages of the SDRF in the gametes can be zero or one, excluding the cases of higher dosages that are possible, although rare, when multivalents are formed. The dosage in progeny individuals derived from self-pollination can thus be either zero (no band), one or two. The parental banding pattern served as a reference to determine these dosages (Figure 2). When the internal distribution of the banding intensities within a progeny individual departed from this parental pattern, the more intense bands could be considered as representing two doses, while the less intense bands could be considered as representing a single dose. Some ambiguity persisted when the intensity distribution among the bands present with a given enzyme was similar to that in the parent; in this case, another restriction enzyme that revealed some

Table 1 Resolution of the 11 BNL12.06 alleles by restriction enzymes HindIII, DraI and SstI

\begin{tabular}{|c|c|c|c|c|c|c|c|c|c|c|c|}
\hline \multirow[t]{2}{*}{ Restriction enzyme } & \multicolumn{11}{|c|}{ Allele } \\
\hline & 1 & 2 & 3 & 4 & 5 & 6 & 7 & 8 & 9 & 10 & 11 \\
\hline HindIII & & & & $\times$ & & $x$ & $x$ & $x$ & & $x$ & \\
\hline DraI & $x$ & $x$ & $\times$ & $x$ & $x$ & $x$ & $x$ & $\times$ & $x$ & & $x$ \\
\hline SstI & & & $x$ & & $x$ & & & & $x$ & & $x$ \\
\hline
\end{tabular}




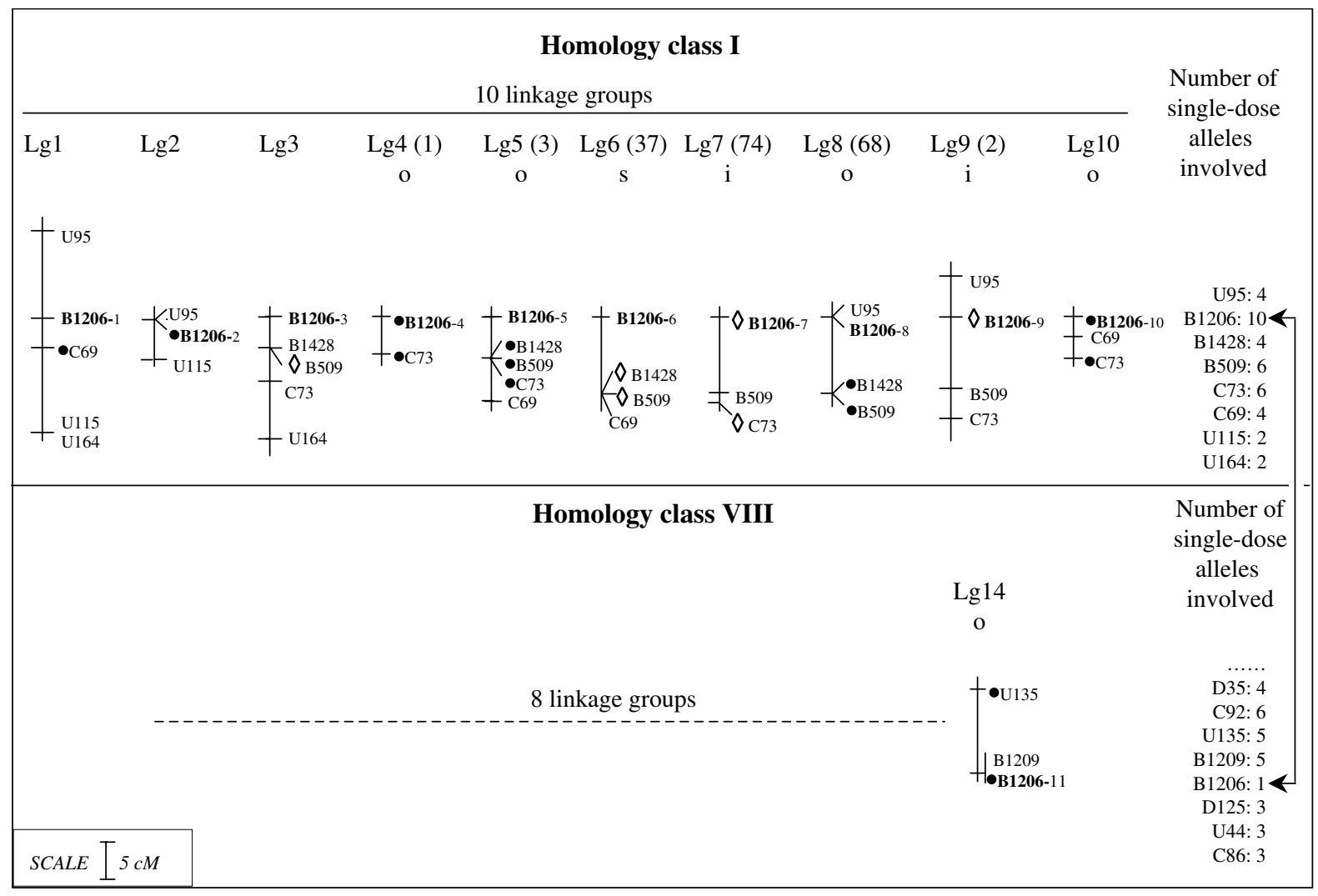

Figure 1 Distribution of the 11 simplex alleles corresponding to the probe BNL12.06 into eleven linkage groups (Lg) according to the map of Grivet $e$ al (1996). On the basis of the loci (corresponding to probes) in common, 10 linkage groups appear homo(eo)logous and are classified into HC I, whereas the 11th one is part of another HC (VIII) made of eight Lg. The BNL12.06 alleles are indicated in bold characters and the nomenclature of the alleles used in the text is indicated. The RFLP probe designation is abbreviated: B stands for BNL, C for SSCIR, D for CDSR, S for SG and U for UMC. The species origin of the alleles on the Lg is indicated when known: a black dot represents S. officinarum and a white losange S. spontaneum. The correspondence of the Lg to those described by Hoarau et al (2001) on the basis of AFLPs is indicated when known. Both RFLP and AFLP marker specificity enable assigning a tentative species origin to some of the Lg: o for S. officinarum, s for S. spontaneum and i for interspecific.

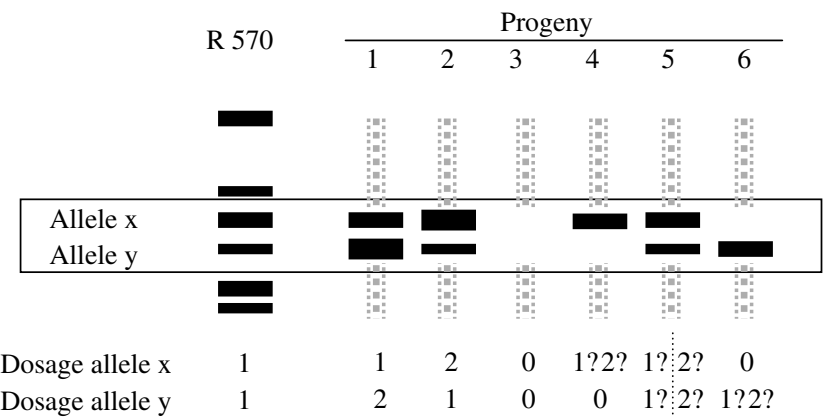

Figure 2 Mode of interpretation of allele dosage in the progeny on the basis of banding intensity patterns. RFLP patterns typically display five to 10 bands with variable intensities. Taking the bands two by two (bands $x$ and $y$ in the example shown), the comparison of the relative intensities with the parental cultivar R570 enables determination of the doses when they are null (progeny 3,4 or 6 ) or uneven (progeny 1 and 2). Thanks to the large number of two by two comparisons, it is generally possible to determine the dosage for all alleles for all progeny individuals.

of the same alleles together with others could indicate the actual dosage (if the intensity distribution departed from the parental one). Altogether, only three individuals were left with some ambiguity; one had six alleles present in either one or two copies, the other two had seven. This analysis thus enabled the description of most individuals by the number of doses $(0,1$ or 2$)$ of all 11 alleles present.

Allele specificity, that is, whether the chromosome segments monitored here are derived from S. officinarum or S. spontaneum, was assessed on the basis of the apparent species origin of the RFLP markers, as determined by Grivet et al (1996), or of the AFLP markers, as determined by Hoarau et al (2001), when the latter could be connected to the RFLP map, directly or after bridging allowed by the study of Rossi et al (2003). The occurrence of several markers close to one another on the genetic map, which displayed a similar species origin, was considered conclusive for the origin of the segment. In the six cases where correspondence with the AFLP groups was established, the specificity analysis conducted by Hoarau et al (2001) could be used and the species origin of a large portion, if not the whole chromosome, could be considered. This resulted in the tentative assignment of an S. officinarum origin to five chromosomes (I-4, I-5, I-8, I-10 of group I and VIII-14 of group VIII, using the nomenclature as described by Grivet et al (1996), an $S$. spontaneum origin to one (I-6 of group I) and a mixed origin to two (I-7 and I-9 of group I, respectively, double 
and simple interspecific recombinants), the rest being of unclear origin.

The analysis of chromosome affinities first considered only bivalent pairing. The estimation of the frequency of pairing between two chromosomes bearing two alleles was performed using a maximum likelihood method. Assume $p_{i j}$ is the frequency of pairing (and $1-p_{i j}$ the frequency of nonpairing) between the two chromosomes that bear alleles $A_{i}$ and $A_{j}$. When these two chromosomes pair together (frequency $p_{i j}$ ), each gamete receives one dose of either allele $A_{i}$ or allele $A_{j}$; when there is no pairing between these two chromosomes (frequency $\left.1-p_{i j}\right)$, but each of them pair to another one, each gamete can receive either allele $A_{i}$, allele $A_{j}$, both of them or neither of them in a similar frequency of $1 / 4$. The frequency distribution of the gametic genotypes can thus be described as a function of $p_{i j}$ and can be used to derive the expected frequencies of the various zygotic genotypes in the progeny, assuming random combination between the male and female gametes. This is developed in Table 2. The frequency of the nine possible zygotic combinations in the whole progeny provides nine equations involving $p_{i j}$ as the sole variable. Knowing the expected and observed frequencies of the different genotypes, a likelihood equation, $L\left(p_{i j}\right)$, can be used to determine the most likely value for $p_{i j}$, as well as the standard deviation of this estimation (Fisher and Balmukand, 1928).

$$
\begin{aligned}
L\left(p_{i j}\right)= & {\left[\frac{(r+s+t+u+v+w+x+y+z) !}{(r ! s ! t ! u ! v ! w ! x ! y ! z !)}\right] } \\
& \times f\left(p_{i j}\right)^{r+z} g\left(p_{i j}\right)^{s+t} h\left(p_{i j}\right)^{u+v} m\left(p_{i j}\right)^{w} n\left(p_{i j}\right)^{x+y}
\end{aligned}
$$

For each pair of alleles, the most likely pairing frequency, $p$, corresponds to the value maximizing the likelihood formula; for practical reasons, it is the logarithm of this formula that is used to determine the most likely pairing frequency, so that $(\log (L(p)))^{\prime}=0$. The result of this exercise is a full table of pairing frequency estimates between the chromosomes that bear the alleles scored.

The patterns expected in case of simple behaviors are given in Table 3, taking an example with 10 alleles. In case of polysomy (Table 3a), it is expected that all pairing frequencies will be $11.1 \%$ (1/9), each chromosome having an equal probability of pairing with any of the other nine. In case of disomy (Table 3b), each chromosome will display pairing frequencies of $100 \%$ with a specific counterpart, and 0 with all the other eight. The

Table 2 Expected frequency of the nine possible genotypes for a couple of simplex alleles, $A_{i}$ and $A_{j}$, in a self progeny

\begin{tabular}{llc}
\hline Genotype & Theoretical frequency & $\begin{array}{c}\text { Observed } \\
\text { frequency }\end{array}$ \\
\hline$[----]$ & $f(p)=(1-p)^{2} / 16$ & $r$ \\
{$\left[A_{i^{-}-}-\right]$} & $g(p)=p(1-p) / 4+(1-p)^{2} / 8$ & $s$ \\
{$\left[--A_{j-}\right]$} & $g(p)=p(1-p) / 4+(1-p)^{2} / 8$ & $t$ \\
{$\left[A_{i} A_{i^{-}}-\right]$} & $h(p)=p^{2} / 4+(1-p)^{2} / 16+p(1-p) / 4$ & $u$ \\
{$\left[--A_{j} A_{j}\right]$} & $h(p)=p^{2} / 4+(1-p)^{2} / 16+p(1-p) / 4$ & $v$ \\
{$\left[A_{i}-A_{j}\right]$} & $m(p)=p^{2} / 2+(1-p)^{2} / 4+p(1-p) / 2$ & $w$ \\
{$\left[A_{i} A_{i} A_{j}\right]$} & $n(p)=p(1-p) / 4+(1-p)^{2} / 8$ & $x$ \\
{$\left[A_{i}-A_{j} A_{j}\right]$} & $n(p)=p(1-p) / 4+(1-p)^{2} / 8$ & $y$ \\
{$\left[A_{i} A_{i} A_{j} A_{j}\right]$} & $f(p)=(1-p)^{2} / 16$ & $z$ \\
\hline
\end{tabular}

$p$ is the pairing frequency of the chromosomes bearing alleles $A_{i}$ and $A_{j}$ at meiosis. possible existence of univalents or trivalents (more complex patterns with higher range multivalents were not considered) is considered in Table 3c:

- a chromosome systematically left as a univalent at meiosis (allele 1 in Table $3 \mathrm{c}$ ) would yield $p_{i j}$ values of 0 in all combinations; the sum of the $p_{i j}$ for the corresponding allele would also be 0 , in contrast with a value of $100 \%$ for a bivalent;

- a set of three chromosomes systematically involved in a trivalent (alleles 2, 3 and 4 in Table 3c) would yield mutual $p_{i j}$ values close to $33.3 \%(1 / 3)$ and values close to 0 in all other combinations; the sum for a given allele would be close to $66.7 \%$, in contrast with a value of $100 \%$ for a bivalent.

In order to analyze the differentiation among the homologous chromosomes, the pair-wise pairing frequencies $p_{i j}$ were used as a measure of similarity. The complements to 1 (ie $1-p_{i j}$ ) were taken as a distance and used to run a factor analysis (StatSoft, 1997). The distribution of the chromosomes along the three major axes was used to summarize the mutual affinities. It also served as a basis to derive and test quantitative features, such as the number of univalents, bivalents or trivalents.

\section{Results}

\section{Marker segregations}

The raw data consist of a matrix of scores of 0,1 or 2 representing the dosages of the 11 alleles for 236 progeny individuals and the dosages of allele 1 to allele 10 for an additional 46 individuals. The respective segregations of the 11 alleles are given in Table 4 . The frequency of transmission of the various alleles ranges from 46 to $54 \%$, with the exception of allele 8 that appears with a frequency of $61 \%$. In several cases (alleles 4, 6, 8 and $10)$, the distribution of the doses departs from that expected in case of identical allele frequencies in the female and male gametes and subsequent random assortment. Such patterns can be explained by differential male and female gametic selection, as is often found in aneuploids (Khush, 1973). The most striking deviation is again found for allele 8. Beyond the individual particularities, the overall pattern of segregation shows an average allele transmission rate equal to the expected $50 \%$.

\section{Pairing frequency estimates}

The pair-wise allele comparisons yielded the most likely pairing frequencies as given in Table 5 . The precision of these values is relatively low, since the standard deviation for each estimate is close to $6 \%$, while many of these estimates are lower than $10 \%$. However, the total range of values spans more than six standard deviations, meaning that many differences among the pairing frequencies are highly significant. The $p_{i j}$ values are comprised between 0 and 39.1\%. Null values highlight couples of chromosomes that never pair together. Values higher than the $10 \%(1 / 10)$ expected from random assortment identify couples that tend to pair preferentially. Although this preferential (more frequent than random) pairing is observed often, there is no case of systematic pairing, which would be identified by a value of $100 \%$. The total of the values for a given allele should 
Table 3 Pairing frequencies (\%) among 10 chromosomes expected from various meiotic assortment patterns

\begin{tabular}{|c|c|c|c|c|c|c|c|c|c|c|c|}
\hline Chromosome & 1 & 2 & 3 & 4 & 5 & 6 & 7 & 8 & 9 & 10 & Total \\
\hline \multicolumn{12}{|c|}{ (a) Polysomy: five random bivalents among the 10 chromosomes } \\
\hline 1 & - & & & & & & & & & & \\
\hline 2 & 11.1 & - & & & & & & & & & \\
\hline 3 & 11.1 & 11.1 & - & & & & & & & & \\
\hline 4 & 11.1 & 11.1 & 11.1 & - & & & & & & & \\
\hline 5 & 11.1 & 11.1 & 11.1 & 11.1 & - & & & & & & \\
\hline 6 & 11.1 & 11.1 & 11.1 & 11.1 & 11.1 & - & & & & & \\
\hline 7 & 11.1 & 11.1 & 11.1 & 11.1 & 11.1 & 11.1 & - & & & & \\
\hline 8 & 11.1 & 11.1 & 11.1 & 11.1 & 11.1 & 11.1 & 11.1 & - & & & \\
\hline 9 & 11.1 & 11.1 & 11.1 & 11.1 & 11.1 & 11.1 & 11.1 & 11.1 & - & & \\
\hline 10 & 11.1 & 11.1 & 11.1 & 11.1 & 11.1 & 11.1 & 11.1 & 11.1 & 11.1 & - & \\
\hline Total & 100 & 100 & 100 & 100 & 100 & 100 & 100 & 100 & 100 & 100 & 1000 \\
\hline \multicolumn{12}{|c|}{ (b) Disomy: five bivalents involving chromosomes $(1,2),(3,4),(5,6),(7,8),(9,10)$} \\
\hline 1 & - & & & & & & & & & & \\
\hline 2 & 100 & - & & & & & & & & & \\
\hline 3 & 0 & 0 & - & & & & & & & & \\
\hline 4 & 0 & 0 & 100 & - & & & & & & & \\
\hline 5 & 0 & 0 & 0 & 0 & - & & & & & & \\
\hline 6 & 0 & 0 & 0 & 0 & 100 & - & & & & & \\
\hline 7 & 0 & 0 & 0 & 0 & 0 & 0 & - & & & & \\
\hline 8 & 0 & 0 & 0 & 0 & 0 & 0 & 100 & - & & & \\
\hline 9 & 0 & 0 & 0 & 0 & 0 & 0 & 0 & 0 & - & & \\
\hline 10 & 0 & 0 & 0 & 0 & 0 & 0 & 0 & 0 & 100 & - & \\
\hline Total & 100 & 100 & 100 & 100 & 100 & 100 & 100 & 100 & 100 & 100 & 1000 \\
\hline \multicolumn{12}{|c|}{$\begin{array}{l}\text { (c) Composite pattern: } 1 \text { ( (univalent with chromosome 1)+1III (trivalent with chromosomes 2, 3, 4)+3II (random bivalents among the six chromosomes } 5 \text {, } \\
7,8,9,10)\end{array}$} \\
\hline 1 & - & & & & & & & & & & \\
\hline 2 & 0 & - & & & & & & & & & \\
\hline 3 & 0 & 33.3 & - & & & & & & & & \\
\hline 4 & 0 & 33.3 & 33.3 & - & & & & & & & \\
\hline 5 & 0 & 0 & 0 & 0 & - & & & & & & \\
\hline 6 & 0 & 0 & 0 & 0 & 20.0 & - & & & & & \\
\hline 7 & 0 & 0 & 0 & 0 & 20.0 & 20.0 & - & & & & \\
\hline 8 & 0 & 0 & 0 & 0 & 20.0 & 20.0 & 20.0 & - & & & \\
\hline 9 & 0 & 0 & 0 & 0 & 20.0 & 20.0 & 20.0 & 20.0 & - & & \\
\hline 10 & 0 & 0 & 0 & 0 & 20.0 & 20.0 & 20.0 & 20.0 & 20.0 & - & \\
\hline Total & 0 & 66.7 & 66.7 & 66.7 & 100 & 100 & 100 & 100 & 100 & 100 & 800 \\
\hline
\end{tabular}

Table 4 Dosage segregation of the 11 BNL12.06 RFLP alleles in a self progeny of cultivar 'R570' constituted of 236 individuals

\begin{tabular}{|c|c|c|c|c|c|c|c|}
\hline \multirow[t]{2}{*}{ Allele } & \multirow[t]{2}{*}{ Chromosome } & \multicolumn{3}{|c|}{ Dosage } & \multirow[t]{2}{*}{ Allele frequency (\%) } & \multicolumn{2}{|c|}{ Distribution } \\
\hline & & 0 & 1 & 2 & & $\chi_{1}^{2}$ & $\chi_{2}^{2}$ \\
\hline 1 & I-1 & 60 & 132 & 44 & 47 & 0.54 & 5.49 \\
\hline 2 & $\mathrm{I}-2$ & 66 & 117 & 53 & 47 & 0.36 & 1.45 \\
\hline 3 & I-3 & 67 & 119 & 50 & 46 & 0.61 & 2.47 \\
\hline 4 & I-4 & 45 & 149 & 42 & 49 & 0.02 & $16.36^{* *}$ \\
\hline 5 & I-5 & 46 & 126 & 64 & 54 & 0.69 & 3.83 \\
\hline 6 & I-6 & 62 & 98 & 76 & 53 & 0.42 & $8.44^{*}$ \\
\hline 7 & I-7 & 62 & 108 & 66 & 51 & 0.02 & 1.83 \\
\hline 8 & I-8 & 56 & 71 & 109 & 61 & $5.95^{*}$ & $61.25^{* *}$ \\
\hline 9 & I-9 & 56 & 130 & 50 & 49 & 0.08 & 2.75 \\
\hline 10 & I-10 & 54 & 136 & 46 & 48 & 0.14 & $6.03^{*}$ \\
\hline 11 & VIII-14 & 48 & 130 & 58 & 52 & 0.21 & 3.29 \\
\hline
\end{tabular}

Nomenclature follows Grivet et al (1996).

$\chi_{1}^{2}: p$ (conformity to a $50 \%$ allele transmission), $1 \mathrm{df}$.

$\chi_{2}^{2}: p$ (random gamete assortment), $2 \mathrm{df}$.

${ }^{*}$ Significant at the $5 \%$ level; ${ }^{* *}$ significant at the $1 \%$ level.

be $100 \%$ if it were involved in a bivalent, irrespective of the specificity of the association. Lower values indicate either pairing with heterologous chromosomes, no pairing (univalent) or pairing in a trivalent. The total ranges from 9.3 to $98.1 \%$, suggesting that some chromosomes are more often involved in pairing with the others. 
Table 5 Maximum likelihood estimates for the pairing frequencies $p_{i j}$ (percent) between chromosomes of HC I as revealed from allele segregation patterns at locus BNL12.06

\begin{tabular}{|c|c|c|c|c|c|c|c|c|c|c|c|c|}
\hline Allele & 1 & 2 & 3 & 4 & 5 & 6 & 7 & 8 & 9 & 10 & 11 & Total \\
\hline Origin $^{\mathrm{a}}$ & $u$ & $u$ & $u$ & $o$ & $o$ & $s$ & $i$ & $o$ & $i$ & $o$ & $o$ & \\
\hline 2 & 27.4 & & & & & & & & & & & \\
\hline 3 & 39.1 & 0.0 & & & & & & & & & & \\
\hline 4 & 0.0 & 4.4 & 1.1 & & & & & & & & & \\
\hline 5 & 0.0 & 12.4 & 12.3 & 0.0 & & & & & & & & \\
\hline 6 & 1.9 & 13.2 & 15.6 & 4.4 & 18.0 & & & & & & & \\
\hline 7 & 6.6 & 13.3 & 9.2 & 11.6 & 22.5 & 22.4 & & & & & & \\
\hline 8 & 3.2 & 8.6 & 1.1 & 13.5 & 0.0 & 0.5 & 4.0 & & & & & \\
\hline 9 & 0.8 & 0.0 & 9.3 & 4.5 & 8.1 & 7.7 & 2.6 & 6.0 & & & & \\
\hline 10 & 2.8 & 3.8 & 7.5 & 26.3 & 0.0 & 5.5 & 1.4 & 23.0 & 0.0 & & & \\
\hline $11^{\mathrm{b}}$ & 0.0 & 0.0 & 2.9 & 1.6 & 4.2 & 0.0 & 0.0 & 0.6 & 0.0 & 0.0 & & \\
\hline Total 1-11 & 81.8 & 83.1 & 98.1 & 67.4 & 77.5 & 89.2 & 93.6 & 60.5 & 39.0 & 70.3 & 9.3 & 811.9 \\
\hline Total 1-10 & 81.8 & 83.1 & 95.2 & 65.8 & 73.3 & 89.2 & 93.6 & 59.9 & 39.0 & 70.3 & - & 751.2 \\
\hline
\end{tabular}

The standard deviation (percents) is between 5.0 and 6.0 for estimation with 282 individuals.

aSuspected origin of chromosome segment, either S. spontaneum (s), S. officinarum (o) interspecific (i) or unclear (u) (9, 18).

bThe estimations for allele 11 were performed on the basis of 236 individuals only.

Allele 11 is actually an outlier with its value of $9.3 \%$, the second lowest value being $39.0 \%$. This distinctiveness is congruent with its linkage, with a range of probes involved in a separate HC. On this basis, it is possible to conclude that it is borne by a chromosome that is not part of the HC under analysis, and to exclude it from subsequent data interpretation. This led to the second line of totals in Table 5.

The totals for one allele range from 39.0 to $95.2 \%$. The mean of the total for the 10 alleles is $75.1 \%$ (Table 5); this figure, compared to $100 \%$, characterizes a global deviation from a typical $5 \times 2$ bivalent meiosis caused by particular cases such as univalents or trivalents.

\section{Specific chromosomal affinities}

The differentiation within the population of chromosomes is illustrated by the results of the factor analysis. The first three axes have eigenvalues of 23,18 and $14 \%$, respectively. The distribution of the chromosomes along these axes is summarized in Figure 3. One chromosome lies apart from the other ones along axis 3. It is I-9, which has a clearly interspecific origin; it is characterized by generally low pairing frequencies with all its counterparts, as illustrated by its total pairing frequency of $39 \%$. Then a group of three chromosomes is isolated. They are chromosomes I-8, I-4 and I-10, which probably have a pure S. officinarum origin. They have a rather low total pairing frequency, with an average of $65 \%$, of which the largest part $(42 \%)$ represents pairing among the three chromosomes. The other six chromosomes, whose putative origins are S. officinarum (I-5), S. spontaneum (I-6), interspecific (I-7) or unclear (I-1, I-2 and I-3), share a rather high average total pairing frequency $(86 \%)$ with $74 \%$ pairing with each other. Among the six, I-1, I-2 and I-3 display a peculiar pattern: I-1 displays a strong preferential pairing with I-2 (27.4\%) and I-3 (39.1\%), while there is no pairing $(0 \%)$ between I-2 and I-3.

\section{Chromosome assortment pattern}

The simplest typical meiotic picture to represent this distribution is: I-9 tending to form a univalent; I-4, I-8 and I-10 tending to form a trivalent or one bivalent and

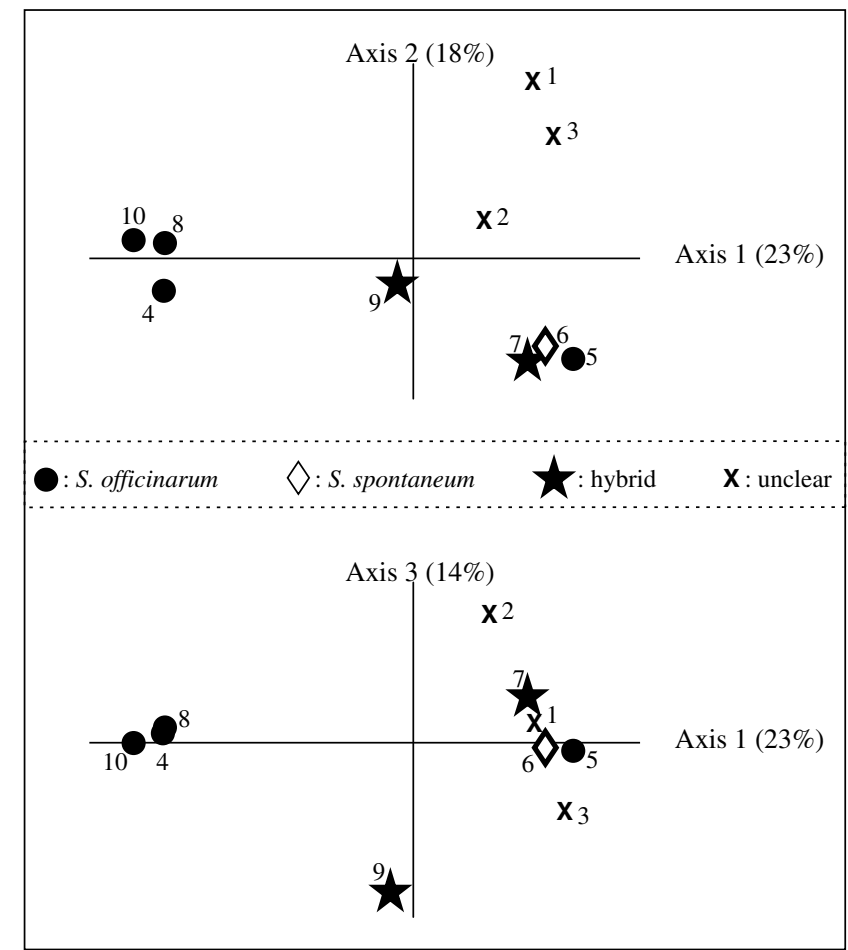

Figure 3 Distribution of 10 alleles representing 10 interacting chromosomes in planes $(1,2)$ and $(1,3)$ of a factor analysis that summarizes the matrix of distances $\left(1-p_{i j}\right)$, where $p_{i j}$ is the frequency of pairing estimated from 282 individuals, as given in Table 5. The species origin of the chromosome segments is indicated.

one univalent; I-1 tending to form a bivalent with I-2 or $\mathrm{I}-3$; and the rest (I-2 or I-3, I-5, I-6 and I-7) forming two bivalents. The translation of various assortment hypotheses into distributions of total allele doses by individual is given in Table 6. The observed distribution is compared to various distributions, corresponding to various meiotic figures that are compatible with the general cytological observations, including: five bivalents (configuration $m_{0}$ ); four bivalents and two univalents $\left(m_{1}\right)$; three bivalents, one trivalent and one univalent 
Table 6 Distribution of the total number of doses found among 282 progeny individuals and comparison with the distribution expected for various meiotic pairing hypotheses $\left(m_{i}\right)$

\begin{tabular}{|c|c|c|c|c|c|c|c|}
\hline \multirow{2}{*}{$\begin{array}{l}\text { Number } \\
\text { of doses }\end{array}$} & \multicolumn{2}{|c|}{ Observed } & \multicolumn{5}{|c|}{ Expected (\%) } \\
\hline & $\mathrm{N}$ & $\%$ & $5 I I\left(m_{0}\right)$ & $\begin{array}{c}4 I I+2 I\left(m_{1}\right) \text { or } \\
1 I I I+3 I I+1 I\left(m_{2}\right)\end{array}$ & $\begin{array}{c}3 I I+4 I\left(m_{3}\right) \text { or } \\
3 I I I+1 I\end{array}$ & $\begin{array}{l}50 \% m_{1} \text { or } m_{2} \\
+50 \% m_{3}\end{array}$ & $\begin{array}{c}75 \% m_{1} \text { or } m_{2} \\
+25 \% m_{3}\end{array}$ \\
\hline 6 & - & 0 & 0 & 0 & 0.4 & 0.1 & 0.0 \\
\hline $7^{a}$ & 4 & 1.4 & 0 & 0 & 3.0 & 1.6 & 0.8 \\
\hline 8 & 21 & 7.4 & 0 & 6.2 & 11.0 & 9.0 & 7.7 \\
\hline 9 & 62 & 22 & 0 & 25.0 & 21.9 & 23.4 & 24.2 \\
\hline 10 & 98 & 34.7 & 100 & 37.5 & 27.3 & 31.8 & 34.5 \\
\hline 11 & 72 & 25.5 & 0 & 25.0 & 21.9 & 23.4 & 24.2 \\
\hline $12^{*}$ & 21 & 7.4 & 0 & 6.2 & 11.0 & 9.0 & 7.7 \\
\hline 13 & 1 & 0.4 & 0 & 0 & 3.0 & 1.6 & 0.8 \\
\hline 14 & 3 & 1.1 & 0 & 0 & 0.4 & 0.1 & 0.0 \\
\hline
\end{tabular}

ancertainty between 7 and 14 for two individuals and between 12 and 6 for one individual.

$\left(m_{2}\right)$; three bivalents and four univalents $\left(m_{3}\right)$. The observed distribution is very close to the $m_{1}$ and $m_{2}$ cases (which are equivalent in terms of dosage distribution), although slightly more deviated towards a distribution expected if more univalents occur ( $m_{3}$ case), as illustrated in the last two columns of Table 6 .

\section{Discussion}

The analysis performed here attempted to describe the mode of chromosome pairing within a given homology class in a sugarcane cultivar. The analytical frame used is complete, since it rests on the survey of (most likely) all alleles at a particular locus, which segregate within a large progeny and whose respective dosages could be determined. The 10 homologues display pairing frequencies ranging from 0 to $40 \%$, and seem differentiated into several subsets:

- one chromosome (I-9) seems often left as a univalent; it is noteworthy that it has a clear interspecific origin, although there is no proof that this is the cause of its isolation at meiosis;

- three chromosomes (I-4, I-8, I-10) seem involved in preferential mutual pairing; whether they assemble into a trivalent or a bivalent plus a univalent cannot be determined from the present data; all probably have a pure $S$. officinarum origin;

- within a subset of six chromosomes, one chromosome (I-1, of unclear origin) seems to preferentially form a bivalent with one of two particular chromosomes (I-2 and I-3, of unclear origin), while the four chromosomes left form two bivalents; this subset includes chromosomes or chromosomal regions originating from the two ancestral species.

The occurrence of univalents or possibly trivalents does not induce a deficit in transmission. Indeed, the chromosomes involved in these figures exhibit the same transmission rate close to $50 \%$ as the others. However, these irregularities induce some variation in the total number of chromosomes transmitted. As a consequence, the distribution of the total dose number per individual is wide but centered around the parental number of 10 . There is no trend towards chromosome loss within this particular HC, but it is noteworthy that about $65 \%$ of the individuals show either more or fewer chromosomes than the parent.
Besides the variation in pairing frequencies observed here, it is known that R570 contains at least two couples of chromosomes that form systematic pairs (Hoarau et al, 2001). Altogether, this remarkable range of affinities among chromosomes somewhat contradicts the traditional view that polyploids tend to classify into allopolyploids with strict bivalent chromosome formation and thus disomic inheritance vs autopolyploids with all homologous chromosomes having equal opportunities to pair at meiosis, leading to polysomic inheritance (Jackson and Jackson, 1996; Sybenga, 1996; Jenczewski and Alix, 2004). These configurations are two extreme cases of polyploids (Wu et al, 2001). Intermediate polyploid models displaying a combination of both allopolyploid and autopolyploid pairing behavior have been suggested by Stebbins (1950) and reported for several polyploid species (Jackson and Jackson, 1996; Allendorf and Danzmann, 1997; Fjellstrom et al, 2001). The locus under study provides additional evidence towards the existence of intermediate polyploid models. The sugarcane cultivar R570 has an artificial origin, for it is derived from man-made interspecific hybrids a few generations ago. However, similar genotypes of spontaneous origin exist within the 'Indian and Chinese sugarcanes', once classified as $S$. barberi and $S$. sinense, respectively, which are now known as derived from a limited number of natural hybridization events between $S$. officinarum and S. spontaneum (D'Hont et al, 2002).

The present study throws light on two other aspects of meiosis in a polyploid genome. First, this system involves locus duplication (if we consider allele 11) and thus a potential bridge between two HC. Such bridges between distinct $\mathrm{HC}$ have been found in low frequency in the genome of R570 (Grivet et al, 1996). They may be the result of genome heterogeneity between the two ancestral species, since these have distinct basic chromosome numbers (D'Hont et al, 1998). They may also be representative of genome structural rearrangements that may occur in polyploids, be they derived from translocations or from ectopic movements associated with transposable element activity (Soltis and Soltis, 1999, 2000). Here, the attachment to two distinct HC resulted in separate pairing dynamics at meiosis. Secondly, the pairing pattern among homologous chromosomes clearly shows that homolog juxtaposition is determined by some features of the individual chromosomes. The mutual affinities appear partly, although incompletely 
determined by the species origin of the chromosomes. With the progress of genetic mapping and allelic resolution along the chromosomes, it will be possible to determine the species origin of the various portions of the chromosomes. When a full picture of these mosaics is available, it will be possible to determine to what extent particular regions of the chromosomes, for example, centromeric or telomeric regions, influence the overall pairing affinities. A generalization of such studies for several HC will give access to a range of situations that will make it possible to test subsequent hypotheses. Therefore, the complex genome of sugarcane will offer material of great interest for basic questions related to the fine mechanisms that govern meiosis.

\section{References}

Al Janabi SM, Honeycutt RJ, McClelland M, Sobral BWS (1993). A genetic linkage map of Saccharum spontaneum L. 'SES 208'. Genetics 134: 1249-1260.

Al Janabi SM, Honeycutt RJ, Sobral BWS (1994). Chromosome assortment in Saccharum. Theor Appl Genet 16: 167-172.

Allendorf FW, Danzmann RG (1997). Secondary tetrasomic segregation of $\mathrm{MDH}-\mathrm{B}$ and preferential pairing of homeologues in rainbow trout. Genetics 145: 1083-1092.

Blanc G, Barakat A, Guyot R, Cooke R, Delseny M (2000). Extensive duplication and reshuffling in the Arabidopsis genome. Plant Cell 12: 1093-1102.

Bremer G (1929). Remarks on the cytology of Saccharum. Facts about Sugar 24: 926-927.

Burner DM (1994). Cytogenetic and fertility characteristics of elite sugarcane clones. Sugar cane 1: 6-10.

Da Silva JAG, Sorrels ME, Burnquist WL, Tanksley SD (1993). RFLP linkage map and genome analysis of Saccharum spontaneum. Genome 36: 782-791.

D'Hont A, Grivet L, Feldmann P, Rao S, Berding N, Glaszmann JC (1996). Characterisation of the double genome structure of modern sugarcane cultivars (Saccharum spp.) by molecular cytogenetics. Mol Gen Genet 250: 405-413.

D'Hont A, Ison D, Alix K, Roux C, Glaszmann JC (1998). Determination of the basic chromosome numbers in the genus Saccharum by physical mapping of RNA genes. Genome 4: 221-225.

D'Hont A, Paulet F, Glaszmann JC (2002). Oligoclonal interspecific origin of 'North Indian' and 'Chinese' sugarcanes. Chrom Res 10: 253-262.

Fisher RA, Balmukand B (1928). The estimation of linkage from the offspring of selfed heterozygotes. J Genet 20: 79-92.

Fjellstrom RG, Breuselinck PR, Steiner JJ (2001). RFLP marker analysis supports tetrasomic inheritance in Lotus corniculatus L. Theor Appl Genet 102: 718-725.

Grivet L, Arruda P (2001). Sugarcane genomics: depicting the complex genome of an important tropical crop. Curr Opin Plant Biol 5: 122-127.

Grivet L, D'Hont A, Roques D, Feldmann P, Lanaud C, Glaszmann JC (1996). RFLP mapping in cultivated sugarcane
(Saccharum spp.): genome organization in a high polyploid and aneuploid interspecific hybrid. Genetics 142: 987-1000.

Hoarau JY, Offmann B, D'Hont A, Risterucci AM, Roques D, Glaszmann JC et al (2001). Genetic dissection of a modern sugarcane cultivar (Saccharum spp.). Part I: Genome mapping with AFLP markers. Theor Appl Genet 103: 84-97.

Jackson RC, Jackson JW (1996). Gene segregation in autotetraploids: prediction from meiotic configurations. Am J Bot 80: 1491-1499.

Jenczewski E, Alix K (2004). From diploids to allopolyploids: the emergence of efficient pairing control genes in plants. Plant Sci 23: 21-45.

Jenkins G, Chatterjee R (1995). Search for a structural component of homology. In: Brandham PE, Bennett MD (eds) Proceedings of Kew Chromosome Conference IV, Royal Botanic Gardens: Kew. pp 365-373.

Khush GS (1973). Cytogenetics of Aneuploids, Academic Press: New York.

Martinez-Perez E, Shaw P, Moore G (2001). The Ph1 locus is needed to ensure specific somatic and meiotic centromere association. Nature 411: 204-207.

Ming R, Liu SC, Lin YR, da Silva J, Wilson J, Braga D et al (1998). Detailed alignment of Saccharum and Sorghum chromosomes; comparative organization of closely related diploid and polyploid genomes. Genetics 150: 1663-1682.

Price S (1963). Cytogenetics of modern sugarcane. Econ Bot 17: 97-106.

Riley R, Chapman V (1958). Genetic control of the cytologically diploid behaviour of hexaploid wheat. Nature 182: 713-715.

Rossi M, Araujo PG, Paulet F, Garsmeur O, Dias VM, Chen H et al (2003). Genomic distribution and characterization of EST-derived resistance gene analogs (RGAs) in sugarcane. Mol Gen Genomics 269: 406-419.

Soltis DE, Soltis SS (1999). Polyploidy: recurrent formation and genome evolution. TREE 14: 348-352.

Soltis DE, Soltis SS (2000). The role of genetic and genomic attributes in the success of polyploids. Proc Natl Acad Sci USA 97: 7051-7057.

Sreenivasan TV (1975). Cytogenetical studies in Saccharum spontaneum L. Proc Indian Acad Sci 81: 131-144.

StatSoft (1997). Statistica for Windows version 5.1. Tulsa, OK, USA.

Stebbins GL (1950). Variation and Evolution in Plants, Columbia University Press: New York.

Stebbins GL (1971). Chromosome Evolution in Higher Plants, Edwards Arnold: London.

Sybenga A (1996). Chromosome pairing affinity and quadrivalent formation in polyploids. Do segmental allopolyploids exist? Genome 39: 1176-1184.

Vision T, Brown D, Tanksley SD (2000). The origins of genomic duplications in Arabidopsis. Science 290: 2114-2117.

Wu KK, Burnquist W, Sorrells ME, Tew TL, Moore PH, Tanksley SD (1992). The detection and estimation of linkage in polyploids using single-dose rstriction fragments. Theor Appl Genet 83: 294-300.

Wu R, Gallo-Meagher M, Littell RC, Zeng ZB (2001). A general polyploid model for analyzing gene segregation in outcrossing tetraploid species. Genetics 159: 869-882. 